\title{
recildunds
}

Revista Científica Mundo de la Investigación y el Conocimiento

Anita María Murillo Zavala a ${ }^{\text {a }}$ Carlos Pedro Marcillo Carvajal ${ }^{\text {b; }}$ Irma Gisella Parrales Pincay ${ }^{c}$; Cristóbal Rolando Barcia Menéndez ${ }^{d}$

Prevalencia de parasitosis en habitantes de 0 a 20 años de la Parroquia El Anegado del Cantón Jipijapa

Prevalence of parasitosis in inhabitants from 0 to 20 years of the Parish of El Anegado del Cantón Jipijapa

Revista Científica Mundo de la Investigación y el Conocimiento. Vol. 3 núm.3, septiembre, ISSN: 2588-073X, 2019, pp. 1294-1302

DOI: $10.26820 /$ recimundo/3.(3).septiembre.2019.1294-1302

URL: http://recimundo.com/index.php/es/article/view/570

Código UNESCO: 3205 Medicina Interna

Tipo de Investigación: Artículo de Revisión

Editorial Saberes del Conocimiento

Recibido: $15 / 05 / 2019$

Aceptado: 23/06/2019

Publicado: 30/09/2019

Correspondencia: director@ recimundo.com

a. Magister en Gerencia y Administración de Salud; Licenciado en Laboratorio Clínico; Universidad Estatal del Sur de Manabí; Jipijapa, Ecuador.

b. Licenciado en Laboratorio Clínico de la Universidad Estatal del Sur de Manabí; Jipijapa, Ecuador.

c. Magister en Gerencia Educativa; Licenciada en Ciencias de la Educación General Básica; Universidad Estatal del Sur de Manabí; Jipijapa, Ecuador.

d. Magister en Diseño y Evaluación de Modelos Educativos; Licenciado en Ciencias de la Salud Especialidad: Laboratorio Clínico; Universidad Estatal del Sur de Manabí; Jipijapa, Ecuador. 


\section{Prevalencia de parasitosis en habitantes de 0 a 20 años de la Parroquia EI Anegado del Cantón Jipijapa}

Vol. 3, núm. 1., (2019)

Anita María Murillo Zavala; Carlos Pedro Marcillo Carvajal; Irma Gisella Parrales Pincay;

Cristóbal Rolando Barcia Menéndez

\section{RESUMEN}

Las parasitosis intestinales constituyen unos de los problemas de salud pública a nivel mundial, cuya prevalencia es mayor en las personas que viven en los países en vías de desarrollo, en donde la presencia y diseminación de estos parásitos están estrechamente relacionadas con las condiciones de saneamiento básico que estén a disposición y los factores socioeconómicos y culturales. El Objetivo fue determinar la prevalencia de parasitosis en residentes de 0 a 20 años de la Parroquia El Anegado del Cantón Jipijapa. El tipo de estudio que se utilizó fue descriptivo, prospectivo, de corte transversal. Se encontró en el total de una muestra de 314 de niños y jóvenes que 178 correspondiente al 56,69\% resultaron positivos a la presencia de parásitos; Los grupos de edad con mayor prevalencia de parasitosis fueron de 6 a 10 años y en segundo lugar de 11 a 15 años. En cuanto a la prevalencia de parásitos, Entamoeba (E,) histolytica se presentó en un 40,13\%, y E. coli en un 25,16\%, Giardia lamblia en un 7,64\% e Himenolepis nana un 0,64\%. Se concluye que la prevalencia de parasitosis en niños y adolescentes de la Parroquia El Anegado del Cantón Jipijapa es muy alta, dado que más de la mitad de las muestras analizadas resultaron positivas. Entre los factores predisponentes identificados se destacan los culturales, por tal motivo se realizaron charlas de prevención para educar

Palabras Claves: Parasitosis; Saneamiento; Epidemiologia; Higiene; Salud. 


\title{
Prevalencia de parasitosis en habitantes de 0 a 20 años de la Parroquia El Anegado del Cantón Jipijapa
}

Vol. 3, núm. 1., (2019)

Anita María Murillo Zavala; Carlos Pedro Marcillo Carvajal; Irma Gisella Parrales Pincay; Cristóbal Rolando Barcia Menéndez

\begin{abstract}
Intestinal parasitic diseases are one of the problems of public health worldwide, whose prevalence is higher in people living in developing countries, where the presence and dissemination of these parasites are closely Related to basic sanitation conditions that are available and socio-economic and cultural factors. The objective was to determine the prevalence of parasitic infections in residents from 0 to 20 years of the parish El flooded of the canton Jipijapa. The type of study used was descriptive, prospective, cross-sectional. It was found in the total of a sample of 314 children and young people that 178 corresponding to $56.69 \%$ were positive to the presence of parasites; The age groups with the highest prevalence of parasitic infections were 6 to 10 years and in second place from 11 to 15 years. As for the prevalence of parasites, Entamoeba (E,) Histolytica was presented in 40.13\%, and E. coli in $25.16 \%$, Giardia lamblia in $7.64 \%$ and Himenolepis nana $0.64 \%$. It is concluded that the prevalence of parasitic infections in children and adolescents of the parish El flooded of the Canton Jipijapa is very high, since more than half of the samples analyzed were positive. Among the predisposing factors identified are the cultural ones, for this reason there were lectures of prevention to educate the population involved on the care and reduction of the risk to this pathology.
\end{abstract}

Key Words: Parasitic Diseases; Sanitation; Epidemiology; Hygiene; Health. 


\section{Prevalencia de parasitosis en habitantes de 0 a 20 años de la Parroquia EI Anegado del Cantón Jipijapa}

Vol. 3, núm. 1., (2019)

Anita María Murillo Zavala; Carlos Pedro Marcillo Carvajal; Irma Gisella Parrales Pincay;

Cristóbal Rolando Barcia Menéndez

\section{Introducción.}

Las parasitosis intestinales constituyen uno de los problemas de salud pública a nivel mundial, cuya prevalencia es mayor en las personas que viven en los países en vías de desarrollo, donde la presencia y diseminación de parásitos está estrechamente relacionada con las condiciones de saneamiento básico que estén a disposición y los factores socioeconómicos y culturales (1).

En Ecuador, según las últimas encuestas realizadas en el 2010 por el Instituto Ecuatoriano de Estadísticas y Censo (INEC) aproximadamente el 62.7\% de los hogares con niños menores de 12 años se encuentran en condiciones de pobreza, siendo las enfermedades intestinales unas de las 10 principales causas de consulta en los centros de salud (2).

En la Parroquia El Anegado, según datos del Plan de Desarrollo y Ordenamiento Territorial (PDyOT) y en diálogo con sus habitantes se pudo evidenciar que la problemática en temas de salud que mayor porcentaje afecta a la parroquia es la parasitosis, causada por falta de un buen servicio de agua potable, alcantarillado sanitario y pluvial, letrinización en comunidades, deficientes conocimientos de higiene, entre otras. Así también se pudo evidenciar la falta de información en temas de parasitosis y prevención de la misma.

La situación actual de los beneficiarios, que según el análisis realizado, hay presencia de parásitos en un 56,7\%, en edades comprendidas de 0 a 20 años, para esto se logró intervenir en una primera fase con la recolección de muestra para posterior a esto tener un diagnóstico mediante examen de laboratorio, además se aplicaron los conocimientos adquiridos en las áreas 


\section{Prevalencia de parasitosis en habitantes de 0 a 20 años de la Parroquia El Anegado del Cantón Jipijapa}

Vol. 3, núm. 1., (2019)

Anita María Murillo Zavala; Carlos Pedro Marcillo Carvajal; Irma Gisella Parrales Pincay; Cristóbal Rolando Barcia Menéndez

de especialidad, encaminados a capacitar y educar a la población de la comunidad de El Anegado.

\section{Antecedentes.}

La parasitosis intestinal sigue siendo un problema de salud pública con prevalencias que pueden llegar a ser muy altas, dependiendo del área geográfica y las condiciones ambientales. Se considera a la población infantil como la población más vulnerable a la parasitosis, donde la probabilidad de infectarse por parásitos aumenta con la edad del niño. Otro de los factores relacionados estaría representado por las características climáticas del área de residencia, períodos de lluvias intensas que favorecen el desarrollo y propagación de las parasitosis, la falta de uso de calzado justificaría la presencia de infecciones causadas por los parásitos identificados. Entre otros factores esta, la ausencia de disponibilidad de agua potable segura para el consumo humano y está vinculada con la presencia de determinados protozoarios (3).

\section{Metodología.}

Esta investigación descriptiva, no experimental, prospectiva, de corte transversal, se realizó en una población de 314 niños y jóvenes de edades comprendidas de 0 a 20 años, residentes en la parroquia Anegado del Cantón Jipijapa, Provincia de Manabí, durante el periodo octubre 2018 - marzo 2019.

Para obtener los datos de diagnóstico de parasitosis se procedió a la realización de estudio de material fecal. Las muestras fueron recolectadas de manera seriada utilizando solución de 


\section{Prevalencia de parasitosis en habitantes de 0 a 20 años de la Parroquia EI Anegado del Cantón Jipijapa}

Vol. 3, núm. 1., (2019)

Anita María Murillo Zavala; Carlos Pedro Marcillo Carvajal; Irma Gisella Parrales Pincay;

Cristóbal Rolando Barcia Menéndez

formalina al 10\% como preservante. Para la participación de los niños (as) y jóvenes, los padres firmaron un consentimiento informado, previo a lo cual se les expuso los objetivos del proyecto, de la fase preventiva, el cuidado en la recolección de la muestra y de la gratuidad del examen realizado.

Para la correcta recolección de las muestras, se les explicó a cada niño y sus padres o representantes de igual manera se les entregó instrucciones escritas, recordando precisión en la recolección; se entregó un recipiente con la inscripción "no derramar", con la debida paleta para la recolección de la muestra, una vez recogidas las muestras se las retiró para el procedimiento de estudio de laboratorio. La evaluación coproparasitaria se realizó en una muestra por cada niño participante. Se empleó la técnica convencional para el examen de heces en fresco por microscopía con solución salina fisiológica y tinción con lugol (4). Una vez terminada la evaluación coproparasitaria, los resultados de laboratorio fueron entregados a los padres de familia de los involucrados, insistiendo en la prevención de parasitosis y la búsqueda de atención médica en caso de padecer parasitosis.

\section{Resultados.}

Se recolectaron un total de 314 muestras de heces, Se pudo determinar que 178 de los sujetos en estudio presentaron parásitos, lo que representa el 56,69\% (tabla 1). 
Prevalencia de parasitosis en habitantes de 0 a 20 años de la Parroquia El Anegado del Cantón Jipijapa

Vol. 3, núm. 1., (2019)

Anita María Murillo Zavala; Carlos Pedro Marcillo Carvajal; Irma Gisella Parrales Pincay; Cristóbal Rolando Barcia Menéndez

Tabla 1. Prevalencia de Parasitados y No Parasitados en niños y jóvenes de la Parroquia El Anegado

\begin{tabular}{lll}
\hline Condición & $\mathbf{n}$ & $\mathbf{\%}$ \\
\hline Parasitados & 178 & 56,69 \\
No parasitados & 136 & 43,31 \\
\hline Total & $\mathbf{3 1 4}$ & $\mathbf{1 0 0 , 0 0}$ \\
\hline
\end{tabular}

Dentro del rango 6 a 10 años se evidenció mayor prevalencia de parásitos en un 37,26\%, en segundo lugar, resultaron las edades comprendidas de 11 a 15 años con un 11,46\%; mientras que entre las edades de 0 a 5 años y 16 a 20 años fue del 7,96\% (Tabla 2).

Tabla 2. Distribución según grupo etario de la infección parasitaria en niños y jóvenes de la Parroquia El Anegado

\begin{tabular}{lllllll}
\hline $\begin{array}{l}\text { Edad } \\
\text { (años) }\end{array}$ & \multicolumn{2}{l}{ Parasitados } & \multicolumn{2}{l}{ No parasitados } & \multicolumn{2}{l}{ Total } \\
\cline { 2 - 7 } & $\boldsymbol{f}$ & $\mathbf{\%}$ & $\boldsymbol{f}$ & $\mathbf{\%}$ & $\mathbf{n}$ & $\mathbf{\%}$ \\
\hline $\mathbf{0 - 5}$ & 23 & 7,32 & 23 & 7,32 & 46 & 14,65 \\
$\mathbf{6 - 1 0}$ & 117 & 37,26 & 89 & 28,34 & 206 & 65,61 \\
$\mathbf{1 1 - 1 5}$ & 36 & 11,46 & 22 & 7,01 & 58 & 18,47 \\
$\mathbf{1 6 - 2 0}$ & 2 & 0,64 & 2 & 0,64 & 4 & 1,27 \\
\hline Total & $\mathbf{1 7 8}$ & $\mathbf{5 6 , 6 9}$ & $\mathbf{1 3 6}$ & $\mathbf{4 3 , 3 1}$ & $\mathbf{3 1 4}$ & $\mathbf{1 0 0 , 0 0}$ \\
\hline
\end{tabular}

En la clasificación por familia de parásitos identificados, se obtuvo que $E$. histolytica está presente en todas las edades, siendo su prevalencia el 40,13\%, seguida; E. coli, en un 25,16\%; en tercer lugar se encuentra el parásito Giardia lamblia en 7,64\%. Y por último Himenolepis nana en un $0,64 \%$ (Tabla 3 ). 


\section{Prevalencia de parasitosis en habitantes de 0 a 20 años de la Parroquia EI Anegado del Cantón Jipijapa}

Vol. 3, núm. 1., (2019)

Anita María Murillo Zavala; Carlos Pedro Marcillo Carvajal; Irma Gisella Parrales Pincay;

Cristóbal Rolando Barcia Menéndez

Tabla 3. Especies parasitarias identificadas según grupos de edad en niños y jóvenes de la

Parroquia El Anegado

\begin{tabular}{lllllllll}
\hline \multirow{2}{*}{ Edad (años) } & \multicolumn{2}{c}{ E. histolytica } & \multicolumn{2}{l}{ E. coli } & \multicolumn{2}{c}{ G. lamblia } & \multicolumn{2}{c}{ H. nana } \\
\cline { 2 - 10 } & F & $\mathbf{\%}$ & F & $\mathbf{\%}$ & F & \% & F & \% \\
\hline $\mathbf{0 - 5}$ & 18 & 5,73 & 5 & 1,59 & 2 & 0,64 & 0 & 0,00 \\
$\mathbf{6 - 1 0}$ & 79 & 25,16 & 57 & 18,15 & 16 & 5,10 & 2 & 0,64 \\
$\mathbf{1 1 - 1 5}$ & 27 & 8,60 & 16 & 5,10 & 6 & 1,91 & 0 & 0,00 \\
$\mathbf{1 6 - 2 0}$ & 2 & 0,64 & 1 & 0,32 & 0 & 0,00 & 0 & 0,00 \\
\hline Total & $\mathbf{1 2 6}$ & $\mathbf{4 0 , 1 3}$ & $\mathbf{7 9}$ & $\mathbf{2 5 , 1 6}$ & $\mathbf{2 4}$ & $\mathbf{7 , 6 4}$ & $\mathbf{2}$ & $\mathbf{0 , 6 4}$ \\
\hline
\end{tabular}

Discusión de resultados.

Los resultados del presente estudio demuestran que de las 314 muestras hay un 56,69\% que presenta parasitosis, y en las cuales el parásito que predomina es el E. histolytica en edades de 6 a 10 años, esta prevalencia de enteroparásitos se debe por las condiciones de saneamiento ambiental inadecuadas en las que vive esta comunidad.

Se concluye que la información que se obtuvo a partir de la presente investigación, refleja condiciones higiénico-sanitarias deficientes en la comunidad bajo estudio.

Se recomienda realizar levantamientos epidemiológicos en las distintas poblaciones aborígenes dentro del país, para así poder profundizar los conocimientos acerca de su situación sanitaria. Es necesario implementar políticas tendientes a mejorar las deficientes condiciones de salud en estas comunidades.

\section{Bibliografía.}

1. Sf V, ML T, FB T. Evolucion de la prevalencia de enteroparasitosis en la ciudad de Talca, 
Prevalencia de parasitosis en habitantes de 0 a 20 años de la Parroquia El Anegado del Cantón Jipijapa

Vol. 3, núm. 1., (2019)

Anita María Murillo Zavala; Carlos Pedro Marcillo Carvajal; Irma Gisella Parrales Pincay; Cristóbal Rolando Barcia Menéndez

Region del Maule, Chile. REv. Chil. Infect. 2010; 27(4).

2. INEC. Instituto Nacional de Estadísticas y Censos. [Online].; 2010 [cited 20171216. Available from: www.inec.gob.ec.

3. Mejía-Delgado M ZAMARMCUTHAL. Factores de riesgo de enteroparasitosis en escolares de la Institucion Educativa Na28629 del caserio Totorilas, Distrito de Guzmango, provincia Contumaza. Rev. Med Trujillo 2014. 2018; 13.

4. D M, M B. Metodos de laboratorios para el diagnsotico de Parasitosis Intestinales Mexico: Interamericano; 1971.

5. Lucero-Garzón TA. Parasitosis Intestinal y Factores de Riesgo en niños. Revista Scielo. 2015; Vol.33(N.2): p. 1/10. 